\title{
Schlegel-Matthies, Kirsten
}

\section{Gesundheit und Selbstverantwortung - Was kann und was sollte gelehrt werden? \\ Haushalt in Bildung \& Forschung 4 (2015) 2, S. 18-30}

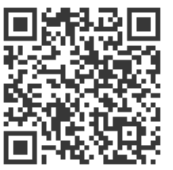

Quellenangabe/ Reference:

Schlegel-Matthies, Kirsten: Gesundheit und Selbstverantwortung - Was kann und was sollte gelehrt werden? - In: Haushalt in Bildung \& Forschung 4 (2015) 2, S. 18-30 - URN:

urn:nbn:de:0111-pedocs-203697 - DOI: 10.25656/01:20369

https://nbn-resolving.org/urn:nbn:de:0111-pedocs-203697

https://doi.org/10.25656/01:20369

in Kooperation mit / in cooperation with:

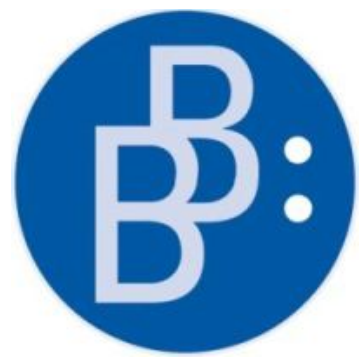

https://www.budrich.de

\section{Nutzungsbedingungen}

Gewährt wird ein nicht exklusives, nicht übertragbares, persönliches und beschränktes Recht auf Nutzung dieses Dokuments. Dieses Dokument ist ausschließlich für den persönlichen, nicht-kommerziellen Gebrauch bestimmt. Die Nutzung stellt keine Übertragung des Eigentumsrechts an diesem Dokument dar und gilt vorbehaltlich der folgenden Einschränkungen: Auf sämtlichen Kopien dieses Dokuments müssen alle Urheberrechtshinweise und sonstigen Hinweise auf gesetzlichen Schutz beibehalten werden. Sie dürfen dieses Dokument nicht in irgendeiner Weise abändern, noch dürfen Sie dieses Dokument für öffentliche oder kommerzielle Zwecke vervielfältigen, öffentlich ausstellen, aufführen, vertreiben oder anderweitig nutzen.

Mit der Verwendung dieses Dokuments erkennen Sie die Nutzungsbedingungen an.

\section{Terms of use}

We grant a non-exclusive, non-transferable, individual and limited right to using this document.

This document is solely intended for your personal, non-commercial use. Use of this document does not include any transfer of property rights and it is conditional to the following limitations: All of the copies of this documents mus retain all copyright information and other information regarding legal protection. You are not allowed to alter this document in any way, to copy it for public or commercial purposes, to exhibit the document in public, to perform, distribute or otherwise use the document in public.

By using this particular document, you accept the above-stated conditions of use.

\section{Kontakt / Contact:}

\section{peDOCS}

DIPF | Leibniz-Institut für Bildungsforschung und Bildungsinformation Informationszentrum (IZ) Bildung

E-Mail: pedocs@dipf.de

Internet: www.pedocs.de

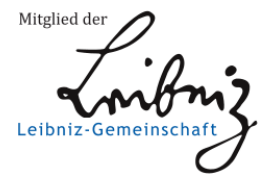


4. Jahrgang Heft 2 2015

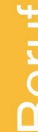

m

(1)

$\frac{\sqrt{2}}{\frac{2}{c}}$

$>$

(1)

(1)

ते

(1)

है

(1)

$\frac{1}{2}$

3

$>$

(1)

$\frac{8}{8}$

$\frac{1}{c}$

है

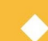

필

$\checkmark$

$\frac{\overline{1}}{\frac{1}{0}}$

है

(1)

(1)
है

$\frac{d}{\frac{c}{c}}$

\section{Haushalt in}

Forschung

Wa(h)re Gesundheit
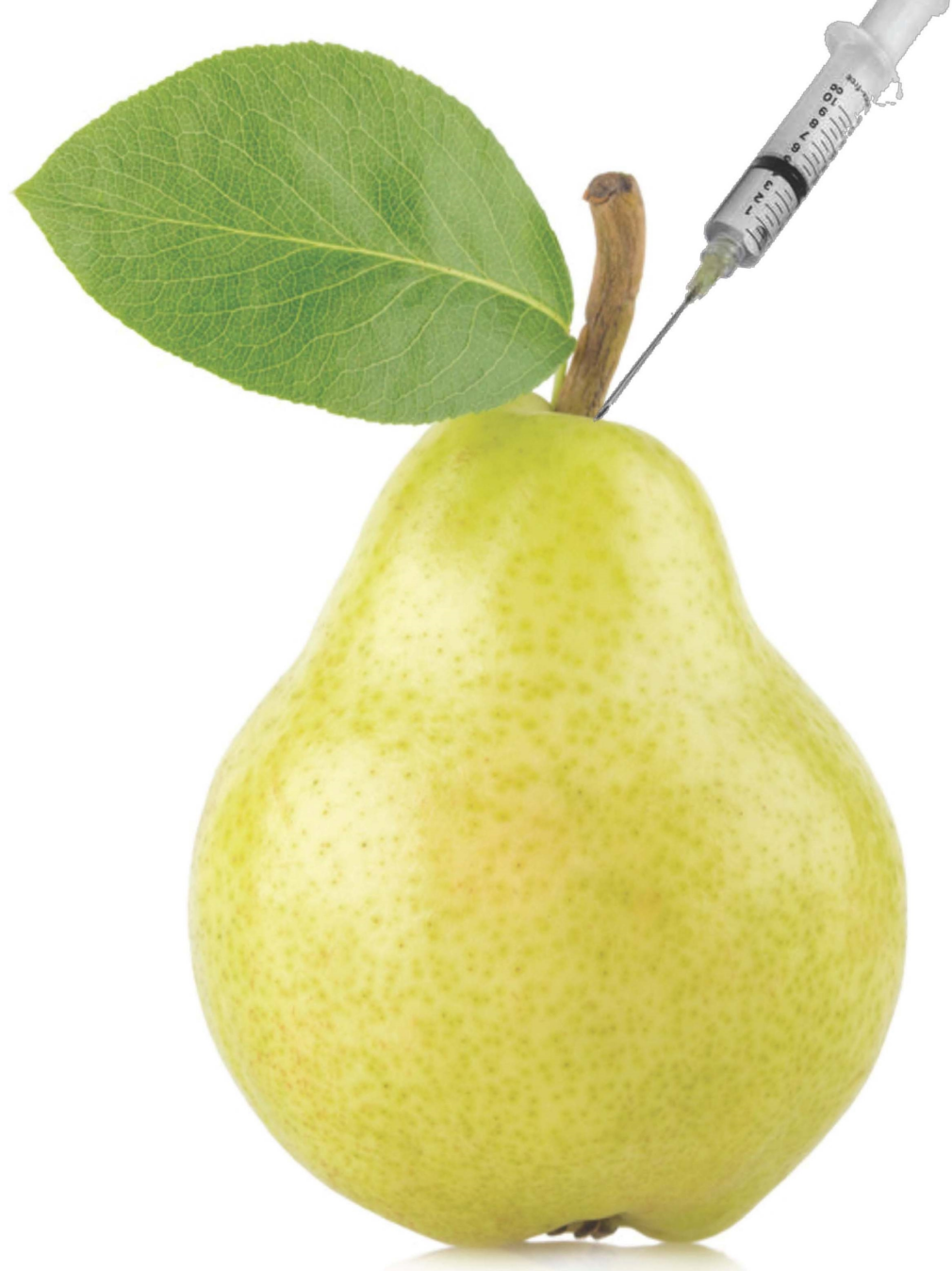
Inhaltsverzeichnis |

Gabriela Leitner

Editorial...... 2

Gabriela Leitner \& Maria Schuh

Ernährungs- und Verbraucherbildung - quo vadis? 3

Kirsten Schlegel-Matthies

Gesundheit und Selbstverantwortung - Was kann und was sollte gelehrt werden?. 18

Angela Häußler

Lässt sich Gesundheit lehren?.

Ute Bender

„Eating Smart“ - Funktionen von Ernährung in der (schulischen)

Gemeinschaftsverpflegung.

Ursula Buchner

Lernwege in der Schulküche. 58

Workshopnachlese 73

Theres Rathmanner

Ernährungsprojekte in Schulen weltweit - ein Blick über den Tellerrand. 86

Michaela Kropatschek

Spielregeln für Werbung im Lebensmittelbereich und in der Schule.

Maya Lucia Joray, Michèle Simone Leuenberger \& Zeno Stanga

Ursachen und Strategien zur Bekämpfung von Übergewicht und Adipositas

bei Kindern und Jugendlichen - aus der Sicht der Schule.

Silke Bartsch \& Werner Brandl

Von der Didaktischen Rekonstruktion zu einer

Didaktik subjektorientierten Lernens und Lehrens.

Rezensionen

Thomas Mohrs

Nachhaltige Lebensstile.... 126

Regine Bigga

Was der Mensch essen darf...... 
Kirsten Schlegel-Matthies

\section{Gesundheit und Selbstverantwortung - Was kann und was sollte gelehrt werden?}

In der Ernährungs- und Verbraucherbildung wird Gesundheit bzw. die Prävention von Erkrankungen oft als Legitimation für Unterrichtsinhalte genannt. Mit der Vermittlung von Wissen über mögliche Erkrankungen und deren Prävention ist jedoch nicht automatisch Lernen über Gesundheit verbunden. Der Beitrag diskutiert, welche Herausforderungen mit „Gesundheit lernen“ in der Ernährungs- und Verbraucherbildung verbunden sind.

Schlüsselwörter: Selbstverantwortung, Prävention, Gesundheit als Anspruch der Gesellschaft

\section{Gesundheit als Bürgerpflicht?}

„Gesundheit ist die erste Pflicht im Leben“"1, schrieb 1895 Oscar Wilde, und tatsächlich scheint Gesundheit heute die erste Bürgerpflicht zu sein: Wer sich nicht regelmäßig sportlich betätigt, Kalorien zählt, viel Obst und Gemüse verzehrt, ausreichend Wasser trinkt, auf Alkohol, Nikotin und andere Drogen rigoros verzichtet - kurz wer möglicherweise einmal die staatliche Krankenversicherung in Anspruch nehmen könnte - verhält sich (gesundheits-)schädlich und erfüllt nicht die Erwartungen, die an ihn bzw. an sie gestellt werden.

Entsprechend gestaltet sich auch die Auseinandersetzung und Thematisierung von Gesundheit in den Medien. So waren beispielsweise am 4. März 2015 bei Google unter dem Stichwort Gesundheit rund 187 Millionen Meldungen zu finden. Dabei wird die gesamte Bandbreite des täglichen Lebens thematisch abgedeckt.

Besonders ausgiebig diskutiert wird der Zusammenhang von Gesundheit und Ernährung (ca. 43 Millionen Meldungen, Stand 04.03.2015). Demgegenüber ist die Anzahl der Meldungen bei den Zusammenhängen von Gesundheit und Bewegung (knapp 27 Millionen Meldungen, Stand 04.03.2015), Alkoholkonsum (fast 9 Millionen Meldungen, Stand 04.03.2015) oder Rauchen (839.000 Meldungen, Stand 04.03.2015) deutlich geringer.

Auch in der Politik wird diesem und den anderen Zusammenhängen besonderes Augenmerk gewidmet. Präventionsprogramme in den Bereichen Ernährung, Bewegung, Tabak- und Alkoholkonsum werden seit Jahren zahlreich gefördert und immer wieder mit unterschiedlichem Fokus neu aufgelegt. Ein Beispiel hierfür ist der Nationale Aktionsplan zur Prävention von Fehlernährung, Bewegungsmangel, Überge- 


\section{Gesundheit und Selbstverantwortung |}

wicht und damit zusammenhängenden Krankheiten - IN FORM (vgl. [www.inform.de]).

So kann es auch nicht verwundern, dass das Bundeskabinett im Dezember 2014 den „Entwurf eines Gesetzes zur Stärkung der Gesundheitsförderung und der Prävention" verabschiedet hat. Im Entwurf werden in $\S 20$, Abs. 2 die folgenden Gesundheitsziele genannt:

1. Diabetes mellitus Typ 2: Erkrankungsrisiko senken, Erkrankte früh erkennen und behandeln,

2. Brustkrebs: Mortalität vermindern, Lebensqualität erhöhen,

3. Tabakkonsum reduzieren,

4. gesund aufwachsen: Lebenskompetenz, Bewegung, Ernährung,

5. gesundheitliche Kompetenz erhöhen, Souveränität der Patientinnen und Patienten stärken,

6. depressive Erkrankungen: verhindern, früh erkennen, nachhaltig behandeln und

7. gesund älter werden“ (Präventionsgesetz - PrävG 2014, S. 8).

Im Mittelpunkt steht die Prävention in Settings bzw. Lebenswelten und hier vor allem die Verhaltensprävention (vor allem die Punkte 1, 3, 4, 5 und 7). Präventionsprogramme, die besonders geeignet sind, „dauerhafte und gesundheitsfördernde Verhaltensänderungen zu bewirken“ (a. a. O., S. 39), sollen außerdem besonders gefördert werden, und die Gesetzliche Krankenversicherung (GKV) kann ihre Bonusprogramme auf zertifizierte Leistungen zur verhaltensbezogenen Prävention weiter verstärken (a. a. O., S. 55). Früherkennungsuntersuchungen für Kinder, Jugendliche und Erwachsene sollen z. B. zu präventionsorientierten Gesundheitsuntersuchungen weiterentwickelt werden. Dabei soll künftig ein stärkeres Augenmerk auf individuelle Belastungen und Risikofaktoren gelegt werden, die zu einer Erkrankung führen können (vgl. BMG, 2014, S. 2). Verhältnisprävention - so der Eindruck - wird zur Nebensache.

Auch die Bundeszentrale für gesundheitliche Aufklärung $(\mathrm{BZgA})$ zielt mit ihrer Definition von Gesundheit in eine ähnliche Richtung, wenn die Bewältigung von inneren und äußeren Anforderungen als Voraussetzung und Grundlage von Gesundheit postuliert wird.

Gesundheit ist das Stadium des Gleichgewichts von Risikofaktoren und Schutzfaktoren, das eintritt, wenn einem Menschen eine Bewältigung sowohl der inneren (körperlichen und psychischen) als auch äußeren (sozialen und materiellen) Anforderungen gelingt. Gesundheit ist gegeben, wenn eine Person sich psychisch und sozial im Einklang mit den Möglichkeiten und Zielvorstellungen und den jeweils gegebenen äußeren Lebensbedingungen befindet. Sie ist ein Stadium, das einem Menschen Wohlbefinden und Lebensfreude vermittelt. (Hurrelmann \& Franzkowiak, 2010)

Diese Definition ist durchaus als problematisch anzusehen, da sie vor allem auf Anforderungen an die Menschen in den privaten Haushalten hinsichtlich ihres Gesundheitsverhaltens zielt. Festgehalten werden kann, dass auch die Diskurse derjenigen, 


\section{Gesundheit und Selbstverantwortung}

die im Gesundheitssystem aktiv sind, in den letzten Jahren von neuen Idealvorstellungen ausgehen, die vor allem „mündige Partner im Gesundheitswesen“ (BMG, 2006, hier zitiert nach Dietrich, 2011, S. 146) erwarten. Einerseits wird damit Individuen im Sinne einer Demokratisierung mehr Mitsprache eingeräumt. Die Akteure im Gesundheitssystem sollten sozusagen aus „Kundenperspektive“ durchaus hinterfragt und nicht als „Halbgötter in Weiß“ betrachtet werden, das Experten-Laien-Gefälle soll demnach verringert werden. Andererseits wird den Patientinnen und Patienten ${ }^{2}$ durch ihre Positionierung als mündige Bürgerinnen und Bürger aber auch eine moralische Verpflichtung für das Gemeinwesen zugewiesen: Gesundheit wird zur Bürgerpflicht! ${ }^{3}$

\section{Gesundheit als Ausdruck des Lebensstils}

Eine solche Verpflichtung zu Gesundheit bzw. zu gesundheitsförderlichen Lebensstilen führt dazu, dass der Prävention besondere Bedeutung zugemessen wird. Darüber hinaus entwickeln sich daraus ein neues Konsumfeld und auch neue Lebensstile. Dies soll in diesem Kapitel dargestellt werden.

Insbesondere im Bereich der Ernährung werden wissenschaftliche Erkenntnisse vielfach als Begründung für verhaltenspräventive Maßnahmen herangezogen. Nach Angaben des Ernährungsberichts 2004 sind über zwei Drittel aller Todesfälle auf Erkrankungen zurückzuführen, bei denen die Ernährung als alleinige Ursache oder als einer von mehreren Faktoren an der Krankheitsentstehung beteiligt ist (vgl. DGE, 2004, S. 94 u. S. 96).

Zahlreiche Studien belegen, dass eine bedarfsgerechte und ausgewogene Ernährungsweise wirksam zur Verhütung zahlreicher Erkrankungen beitragen kann (vgl. z. B. Schacky, 2008; Willett \& Stampfer, 2013). Interessant ist dabei vor allem, ob die Ergebnisse solcher Studien überhaupt und wie wahrgenommen werden und welche Konsequenzen aus den Studien gezogen werden.

In den Medien werden die Ergebnisse wissenschaftlicher Studien allzu oft vereinfacht und auf „Schreckensmeldungen“ reduziert, denn nur eine schlechte Nachricht ist bekanntlich eine Nachricht. In der Zeitung „Die Welt“ vom 9. Februar 2015 findet sich beispielsweise ein Artikel mit der Überschrift „So gefährlich sind Fett, Salz, Zucker und Alkohol" und weiter heißt es:

Sie sind die bösen Vier, die größten Essenssünden, denen wir uns regelmäßig hingeben. Ernährungsforscher haben ausgerechnet, wie viel Gramm lebensnotwendig und wie viel ungesund sind. (Sibbel \& Kirchner, 2015).

Die Verbindung von Essverhalten und Sünde rückt das angeprangerte ,falsche Verhalten" nicht nur in einen religiösen Kontext. Zugleich wird dadurch die Schuld derjenigen überhöht, die sich nicht an die Maßgaben der Wissenschaft halten. Nicht mehr die sieben Todsünden, sondern die „bösen Vier“ führen zur Verdammnis, 


\section{Gesundheit und Selbstverantwortung}

sprich zu Krankheit und Sterben. Absolution kann demnach nur erhalten, wer sich dem Gesundheitsdiktat unterwirft. Das Gesundheitsdiktat der Experten und Expertinnen aus den unterschiedlichen wissenschaftlichen Vereinigungen (genannt werden im Artikel die deutsche Leberstiftung, die DGE, der Berufsverband Deutscher Neurologen, die Deutsche Gesellschaft für Zahnerhaltung, die WHO, die Deutsche Gesellschaft für Hypertonie und Prävention sowie der Berufsverband für Kinder- und Jugendpsychiatrie) soll dann von mündigen (?) Bürgerinnen und Bürgern selbstverantwortlich aufgegriffen und im täglichen Leben umgesetzt werden. So verstanden wird Wissenschaft zum Dogma!

Ein anderer Artikel gibt Tipps für die gesundheitsförderliche Ausgestaltung der Mittagspause: „Wie Sie die Mittagspause für Ihre Gesundheit nutzen“ (rp-online vom 12.02.2015). Hier geht es weniger um das Anprangern von gesundheitsschädlichem Verhalten als vielmehr darum, Gesundheit im Sinne von Leistungsfähigkeit und Produktivität besonders zu fördern.

Gesundheit - das zeigen die wenigen Beispiele deutlich - ist „Mega-Trend“ (Schmidt, 2010, S. 23; vgl. ausführlich Raacke, 2014). Aus solchen Mega-Trends erwachsen einerseits Märkte für entsprechende Konsumgüter und Dienstleistungen und andererseits Anforderungen an die Menschen in ihrer Rolle als Verbraucherinnen und Verbraucher.

\subsection{Gesundheit als Markt}

„Gesundheit ist ein ansteckender Markt“ Raymond Walden ${ }^{1}$

Ein zentrales Argument für präventive Maßnahmen und Gesundheitsförderung im Allgemeinen sind die stetig steigenden Kosten im Gesundheitswesen. Diese beliefen sich im Gesundheitswesen im Jahr 2012 auf mehr als 300 Mrd. Euro (vgl. Statistisches Bundesamt, 2014), das entspricht einem Anteil von 11,3\% des Bruttoinlandsprodukts (vgl. ebd.). Auf den sog. zweiten Gesundheitsmarkt, also auf die Konsumausgaben der privaten Haushalte für „Gesundheit“, die nicht durch die private (PKV) und die gesetzliche Krankenversicherung (GKV) abgedeckt werden (vgl. AG Gesundheitsökonomische Gesamtrechnung, 2014), entfielen davon rund 65 Mrd. Euro. „Falsche“ Lebensstile sollen demnach durch Aufklärung, Information, Bildung und Beratung sowie durch Nudging ${ }^{4}$ (vgl. z. B. Thaler \& Sunstein, 2010) verändert werden.

Auch über Spezialprodukte im Lebensmittelsektor, z. B. bei Glutenunverträglichkeit, Lactoseintoleranz oder Allergien, werden neue Märkte aufgebaut, die den Anbietern Gewinne und den Konsumentinnen und Konsumenten „Gesundheit" versprechen. Und dies geschieht unabhängig davon, ob eine entsprechende Intoleranz oder Unverträglichkeit überhaupt ärztlich diagnostiziert wurde. 


\section{Gesundheit und Selbstverantwortung}

Individuelle Gesundheitsleistungen (IGeL) ergänzen mit immer mehr Angeboten die Leistungen der Krankenkassen, Wellness- und Fitnessprodukte und entsprechende Dienstleistungen werden zunehmend angeboten und nachgefragt. Ärzte sprechen im Zusammenhang mit IGeLeistungen meist nicht mehr von Patientinnen und Patienten sondern von Kundinnen und Kunden oder Klienten. D. h. hier erfolgt eine Rollenzuweisung hin zur Konsumentenrolle. Gesundheit und Krankheit werden damit zum Markt und müssen sich damit den Regeln von Märkten beugen.

Der Gesundheitsmarkt wird - so die Prognosen - stetig weiter wachsen und verspricht für die Gesundheitswirtschaft stetig wachsende Gewinnspannen (vgl. Flexperto.com, 2014), weil gesundheitsfördernde Lebensstile für die privaten Haushalte immer mehr an Bedeutung gewinnen und allgemein das Gesundheitsbewusstsein innerhalb der Bevölkerung steigt. Damit wächst zugleich das Interesse der Gesundheitswirtschaft an neuen Märkten (vgl. Deloitte \& Touche, 2014). E-Health kommt dabei eine besondere Rolle zu, denn Mobilität und Digitalisierung durchdringen alle Lebensbereiche.

Web-basierte Gesundheitsportale, Apps, Mess- und Assistenzsysteme oder digitale Fitness-Tools erobern den Alltag. Laut einer Studie des Beratungs- und Wirtschaftsprüfungsunternehmens (!) Deloitte \& Touche gibt es aktuell mehr als 47.000 „Health Care Apps“ im Apple iTunes Store und mehr als 100.000 mobile „Health Care Apps“ auf verschiedenen anderen Plattformen (vgl. Deloitte \& Touche, 2014, S. 9) - ganz nach dem Motto: „Ein gesunder Mensch ist falsch untersucht"“1.

\subsection{Healthstyle als Distinktionsmerkmal}

„Wer sich heute noch gesund fühlt, muss krank sein“ Gerhard Kocher ${ }^{1}$

Dieser Ausspruch verweist auf das Phänomen des sog. Healthstyle, der als neuer, präventiver Lebensstil für viele Menschen immer mehr an Bedeutung gewinnt. Der Verzehr von Bio-Lebensmitteln, regelmäßige Bewegungs- und Sportaktivitäten sowie immer mehr Wellnessprodukte und -dienstleistungen kennzeichnen diesen Lebensstil, der auf Gesundheit und hier vor allem auf Schlankheit, Leistungsfähigkeit und Kontrolle von Körperfunktionen und Vitaldaten abzielt. Per Smartphone-App werden z. B. die Vitaldaten überwacht und regelmäßig abgerufen, kann der Blutzucker bestimmt oder sogar ein Syphilis- oder HIV-Test durchgeführt werden (vgl. Focus online, 2015; Lifeline, 2015). Die Kenntnis von Normwerten für den ,richtigen“ Gesundheitszustand gehört ebenso zu diesem Lebensstil wie die häufige/regelmäßige Nutzung von Gesundheitsportalen oder die Selbstdiagnose von Glutenunverträglichkeit und/oder Lactoseintoleranz ohne Konsultation oder Diagnose eines Arztes oder einer Ärztin.

Damit verbunden sind aber auch ein abnehmendes Vertrauen in die eigene Körperwahrnehmung und den eigenen Körper, wenn Sich-wohl-Fühlen allein nicht mehr 


\section{Gesundheit und Selbstverantwortung}

ausreicht. Die Vorstellung der Möglichkeit einer Optimierung des eigenen Körpers, des Gesundheitszustandes und letztlich des gesamten Lebens scheint sich zunehmend zu verbreiten, und der Markt reagiert darauf (vgl. Friedrichs, 2013).

Die E-Health-Angebote passen scheinbar genau in die Lebenswelt der „Digital Natives“, denn sie ermöglichen eine immer stärkere Kontrolle der Körperfunktionen und eine langfristige Überwachung des individuellen Fitness- und Gesundheitszustandes. Zum anderen sind sie aber auch im modischen Trend, sie sind innovativ und „,cool“. Wer sie nutzt, zeigt auch, dass er oder sie „dazu“ gehört und sich von denjenigen abgrenzt, die vermeintlich gar nicht oder weniger auf ihre Gesundheit achten.

Folglich kann es für den bzw. die Einzelne zunehmend schwieriger werden, sich diesen Anforderungen zu entziehen, die dann nicht mehr als Handlungsmöglichkeiten, sondern als Handlungszwänge wahrgenommen werden. Ein Abweichen von der Norm (vgl. Raacke, 2014) wird schnell zum persönlichen Versagen und damit letztlich zur Schuld.

„Viele überleben eine gesunde Lebensweise nicht“ (Walter Ludin) ${ }^{1}$. Wenn Gesundheit überwiegend unter dem Aspekt der Kosten oder der Leistungsfähigkeit im Beruf betrachtet wird, dann wird sie in dieser Argumentation zur Leistung des Individuums für die Gesellschaft. Sie ist also nicht mehr Privatsache und Schicksal, sondern eine für die Gemeinschaft zu erbringende Leistung. Wer nicht gesund ist, hat sich demnach nicht entsprechend den Handlungsempfehlungen der Experten und Expertinnen verhalten. Folgt man dieser Argumentation, dann werden aus Kranken nicht nur Opfer ungünstiger Bedingungen, sondern Unterlassungstäter bzw. Unterlassungstäterinnen.

Seitdem Vorsorge für Gesundheit einen immer größeren Stellenwert erhält, wird Krankheit immer weniger als körperlich erfahrbares Phänomen verstanden, das befundet wird, wenn es vorhanden ist. Heute wird Krankheit vielmehr als fehlgeleitete Entwicklung wahrgenommen, die bei früherer oder rechtzeitiger Intervention zu vermeiden gewesen wäre. (Hahn, 2011, S. 30).

Diese Sichtweise macht dann allerdings Gesundheit der Individuen zum gesellschaftlichen und damit zum politischen Problem. Krankheit wird zunehmend verstanden als Ausdruck und Ergebnis von Fehlverhalten. Gesundheit und Krankheit sind aber immer auch gesellschaftlich konstruiert und das, was als gesund oder als krank gilt, verändert sich je nach gesellschaftlicher Situation und Zeit. ${ }^{5}$

Damit verbunden sind gesellschaftliche Zuschreibungen, die zugleich ausgren-

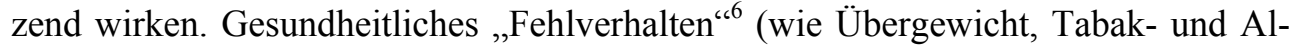
koholkonsum) wird den Angehörigen der sozialen Unterschichten zugeordnet, während gesundheitsorientierte Lebensstile häufiger den leistungsorientierten Eliten zugewiesen werden (vgl. Aronowitz, 2008; Niewöhner, 2008).

Wenn der aktuelle Gesundheitsdiskurs aber dazu genutzt wird, soziale Grenzen aufrecht zu erhalten und deren Verschwimmen zu verhindern, dann müssen Bemü- 


\title{
Gesundheit und Selbstverantwortung
}

hungen der Gesundheitsbildung und -erziehung besonders kritisch betrachtet werden. Darauf soll im folgenden Abschnitt eingegangen werden.

\section{Gesundheit lernen im haushaltsbezogenen Unterricht?}

\author{
„Gesundheitsapostel werden leicht Evangelisten“ \\ Gerhard Uhlenbruck ${ }^{1}$
}

Im haushaltsbezogenen Unterricht wird „Gesundheit“" von vielen Lehrerinnen und Lehrern noch immer und vor allem verstanden als die Vermittlung von ,gesunder Ernährung“. ${ }^{7}$ Der öffentliche Gesundheitsdiskurs wirkt auch auf Lehrpersonen und deren Vorstellungen vom „Fach“ und von den damit verbundenen Aufgaben. ${ }^{8}$ Lehrpersonen orientieren sich z. B. an Schulbüchern, die diesen Diskurs unreflektiert abbilden.

Falsche Verhaltensweisen und Gewohnheiten der Schülerinnen und Schüler - so die Vorstellung - müssen durch die Vermittlung von Wissen über „richtige“, weil gesundheitsförderliche Ernährung sowie die Vermittlung von Wissen über ernährungsmitbedingte Erkrankungen verändert werden. Ob die Schülerinnen und Schüler „gesundheitsriskante“ individuelle Verhaltensweisen und Gewohnheiten haben und ob bzw. warum diese geändert werden sollten, wird dabei von den Lehrpersonen selten infrage gestellt oder reflektiert.

Ernährungsbildung wird nicht nur in Bildungsplänen und Richtlinien, sondern auch von Lehrpersonen im Fach häufig gleichgesetzt mit der Vermittlung von Wissen über ,gesunde“ Ernährung. Schülerinnen und Schüler sollen dann beispielsweise Merkmale einer „falschen Ernährung“ herausarbeiten und daraus z. B. „Handlungsempfehlungen“ für eine Änderung des Ernährungsverhaltens entwickeln. Zielsetzung eines solchen Unterrichts sind die Prävention von Übergewicht und Adipositas sowie von anderen ernährungs(mit)bedingten Erkrankungen und eben die Änderung von „Fehlverhalten“. Im schlechtesten Fall folgt daraus sogar die ausführliche Thematisierung von Erkrankungen wie Gicht, Adipositas, Bluthochdruck.

Vermeintliche Unterstützung erfährt ein solcher Unterricht z. B. durch die Empfehlung zur Gesundheitsförderung und Prävention in der Schule der KMK (2012). In der Empfehlung wird die „Ernährungs- und Verbraucherbildung einschließlich Schulverpflegung“ ausdrücklich in die Liste der zur schulischen Gesundheitsförderung und Prävention benannten Themen und Handlungsfelder aufgenommen (KMK, 2012, S. 5).

Allerdings ist dies noch keine ausreichende Begründung für die im oben genannten Beispiel angeführten Unterrichtsschwerpunkte. Der Bildungswert von Ernährungsthemen muss immer auch fachdidaktisch und pädagogisch begründet und dargelegt werden. Eine Bezugnahme allein auf die entstehenden Kosten für das 


\title{
Gesundheit und Selbstverantwortung |
}

Gesundheitswesen bzw. auf die Möglichkeit einer Erkrankung im späteren Leben kann und darf nicht ausreichend sein.

In der Schule bzw. im schulischen Unterricht innerhalb der Ernährungs- und Verbraucherbildung wird jedoch Gesundheit oft zum Selbstzweck und allein der Verweis darauf, dass es ja schließlich um Gesundheit gehe, tritt an die Stelle der fachdidaktischen Begründung und vor allem der Reflexion der Planungsentscheidungen, ja lässt diese für die beteiligten Lehrpersonen gleichsam unnötig erscheinen.

Hier spiegelt sich die oben dargestellte gesellschaftliche Entwicklung wider, in der gesundheitsförderliche Lebensstile eben auch ein Merkmal der gesellschaftlich ungleichen Positionierung (auch von Schülerinnen und Schülern auf der einen Seite und Lehrpersonen auf der anderen Seite) sind und damit Ex- und Inklusionen legitimieren. Für Lehrpersonen folgt daraus, die Rückbesinnung auf die Aufgaben und Ziele von Schule allgemein und des haushaltsbezogenen Unterrichts im Besonderen nicht aus dem Blick zu verlieren und für die Unterrichtsplanung ernst zu nehmen.

\section{Gesundheitsbildung als Bestandteil der Ernährungs- und Verbraucherbildung}

\author{
„Sei vorsichtig beim Lesen von Gesundheitsbüchern, \\ der kleinste Druckfehler kann dein Tod sein" \\ Mark Twain ${ }^{1}$
}

Ziel der Ernährungs- und Verbraucherbildung bzw. des haushaltsbezogenen Unterrichts ist die Vermittlung von Daseinskompetenzen zur Schaffung von je individueller Lebensqualität ${ }^{9}$ (vgl. z.B. Schlegel-Matthies, 2015). Weder Gesundheit an sich, noch gesunde Ernährung oder nachhaltige Lebensführung, rationale Konsumentscheidungen usw. können und dürfen für sich allein eine ausreichende Legitimierung für die Auswahl von Themen und Inhalten sowie die Festsetzung von Lehr- und Lernzielen sein.

Erforderlich ist vielmehr die Legitimation von Unterrichtsinhalten immer wieder auf der Grundlage der Aufgabe von Schule insgesamt reflexiv zu klären und abzusichern. Der REVIS-Referenzrahmen, der den Bildungsanspruch junger Menschen im Bereich der Ernährungs- und Verbraucherbildung beschreibt, und der didaktische Würfel (vgl. Fachgruppe Ernährungs- und Verbraucherbildung, 2005, S. 30f.) können dabei hilfreich sein, dies allerdings nur, wenn mit dem Verweis auf die Bildungsziele und Kompetenzen des Referenzrahmens nicht lediglich die eigenen (dogmatischen) Vorstellungen begründet werden. Nur so kann sichergestellt werden, dass nicht „Gesundheit lernen“ an die Stelle von „Rezepte zubereiten“ tritt. Es gilt vielmehr, den REVIS-Referenzrahmen wie jedes andere zugrunde gelegte Konzept offen zu legen und hinsichtlich seiner Reichweite und Ausrichtung kritisch zu hinterfragen. 


\section{Gesundheit und Selbstverantwortung}

Für die Gesundheitsbildung im Rahmen der Ernährungs- und Verbraucherbildung bedeutet dies zunächst einmal zu klären, welchen Anteil Gesundheit an einem „guten Leben“ bzw. an der Zielvorstellung Lebensqualität haben kann und welche Anteile davon auf individuelles Verhalten und auf soziale Verhältnisse entfallen. Konkret bedeutet dies, sich mit dem herrschenden Gesundheitsdiskurs kritisch auseinanderzusetzen und ihn auf normative Anteile hin zu hinterfragen. Dabei könnte deutlich werden, dass die Orientierung am vorherrschenden Gesundheitsleitbild „Gesundheit als Leistung des Individuums für die Gesellschaft“ der Zielsetzung des Faches zuwider läuft, wenn sie die Ziele des Unterrichts bestimmt, denn die orientieren sich stärker an der Befähigung des Individuums zur Reflexion dieses Leitbildes Haushaltsbezogener Unterricht hat bei der Anbahnung von Daseinskompetenzen zur Schaffung und Bewahrung von Lebensqualität vor allem die sich eröffnenden Handlungsoptionen in den Blick zu nehmen und diese hinsichtlich ihrer unterschiedlichen Qualitäten mit den Schülerinnen und Schülern zu reflektieren.

Bei der Planung von Lernarrangements sollten deshalb Kompetenzen angebahnt werden, die auch im Bereich Gesundheit den selbstbestimmten und reflektierten Umgang mit sich widersprechenden, mit überfordernden oder mit - aus der Sicht der Einzelnen - nicht nachvollziehbaren Anforderungen ermöglichen. So wird auch die Kompetenzformulierung im REVIS Bildungsziel 2

Die Schülerinnen und Schüler sind bereit und in der Lage, sich mit dem Zusammenhang von Ernährung und Gesundheit auseinanderzusetzen und Verantwortung für sich und andere zu übernehmen. (Fachgruppe Ernährungs- und Verbraucherbildung, 2005, S. 26)

nicht im Sinne des oben beschriebenen Gesundheitskonstrukts verstanden und mit der Frage nach der „Schuld“ bei Fehlverhalten verbunden. Dies wäre z. B. der Fall, wenn - wie erfahrungsgemäß im Unterricht vorkommend - die zehn Regeln der DGE als unhinterfragte Richtschnur des Handelns (gleichsam als die Zehn Gebote der Ernährung) vermittelt werden. Es geht vielmehr darum, Schülerinnen und Schüler zu befähigen, auch diese Empfehlungen für unterschiedliche Kontexte situationsspezifisch einordnen und bewerten zu können. Dafür sind Wissen und Verstehen über Entstehung, Zielsetzung und Reichweite der Empfehlungen, die Kenntnis der zugrunde liegenden Fakten, auch die Kenntnis davon abweichender Empfehlungen, das Abwägen von Informationen, die Einschätzung der jeweiligen Situation usw. nötig. Auch hier gilt: „Nicht die Vermittlung von Gewissheiten ist das Ziel, sondern die Befähigung zu deren Hinterfragung!“" (Schlegel-Matthies, 2013, S. 67).

Gesundheit bzw. gesundheitsförderliche Ernährung würde dann nicht uneingeschränkt als „gut“ an sich vermittelt. Die Vermittlung von gesundheitsförderlichen Handlungsoptionen müsste vielmehr im Kontext von Teilhabe, Mitgestaltung und Selbstbestimmung der Lernenden in der Ernährungs- und Verbraucherbildung betrachtet und bewertet werden (vgl. Klafki, 1996; Schlegel-Matthies, 2013). Damit wird Gesundheit zu einer Perspektive neben anderen, die auch berücksichtigt werden 


\section{Gesundheit und Selbstverantwortung}

kann, und nicht zu einer Anforderung, die durch bzw. über Unterricht eingelöst werden muss.

\section{Anmerkungen}

1 Alle Zitate und Lebensweisheiten zur Gesundheit, soweit nicht anders angegeben, aus [www.aphorismen.de].

2 Die Bezeichnung Patientinnen und Patienten wurde hier gewählt, um deutlich zu machen, dass es hier um Personen geht, die sich mit ihren Beschwerden in ärztliche Behandlung begeben.

3 Vgl. dazu ausführlich Dietrich (2011), die die Zuschreibung von Eigenverantwortung über die gesamte Lebensspanne auch im Falle von Krankheit und die Auswirkungen auf die Krankenversorgung untersucht (Dietrich, 2011).

4 „Nudging“ wird übersetzt als Anstoßen oder Schubsen. Der Ansatz stammt aus der Verhaltensökonomie. Der Begriff wurde von Richard H. Thaler und Cass R. Sunstein in ihrem Bestseller „Nudge. Improving Decisions About Health, Wealth, and Happiness" aus dem Jahr 2008 geprägt. Menschen sollen durch den richtigen Anstoß bzw. die richtigen Rahmenbedingungen von ganz allein zu gewünschten Entscheidungen kommen.

5 Der Bauch eines Mannes im 19. Jahrhundert signalisierte Wohlstand und Gesundheit, die Schlankheit eines Mannes aus der Unterschicht, eines „Hungerleiders“ hingegen Armut und einen schlechteren Gesundheitszustand. Heute sind die Konnotierungen genau umgekehrt: Beleibtheit ist Zeichen für die Zugehörigkeit zur sozialen Unterschicht und fehlendes Gesundheitsbewusstsein, Schlankheit Zeichen für Gesundheitsorientierung, Leistungsfähigkeit und Zugehörigkeit zur sozialen Oberschicht.

6 Bereits im Begriff Fehlverhalten kann eine Problematisierung von sog. riskanten Lebensstilen vermutet und unterstellt werden. Ausführlich zur Diskussion dazu: Hahn, 2011, S. 42f.

7 Eine erfreuliche Ausnahme ist: Leutnant, S. (Hrsg.) (2014). Plan L. Leben bewusst gestalten. Ernährung - Konsum - Gesundheit. Paderborn: Schöningh Verlag.

8 Darüber hinaus kommen schon die Schülerinnen und Schüler mit polarisierenden Vorstellungen zu ,gesund“ oder ,lecker“ in den Unterricht. Dies verstärkt zugleich die unreflektierte Übernahme von normativen Setzungen, die sich hinter der Vermittlung von ,gesunder“ Ernährung finden lassen.

9 Die Diskussion um „richtiges“ Haushalten und den Sinn des richtigen Haushaltsens wird seit der Antike geführt. Immer geht es um die Frage der Definition und Herstellung von Lebensqualität. Lediglich in den 1970er Jahren wurde mit der Wendung zur naturwissenschaftlichen Grundlegung des Faches in Deutschland die Diskussion um normative Implikationen infrage zu stellen. In der aktuellen Diskussion werden keinerlei Vorgaben für die Lebensgestaltung und die daraus abgeleitete 


\section{Gesundheit und Selbstverantwortung}

Lebensqualität mehr gemacht. Der Fokus liegt vielmehr auf der Anbahnung und Diskussion notwendiger Kompetenzen und vor allem auf der Reflexion darüber. Vgl. zur Geschichte ausführlich Schlegel-Matthies, 2015 und Tornieporth, 1977 sowie zur aktuellsten Diskussion Hirschfelder, Ploeger, Rückert-John und Schönberger, 2015.

\section{Literatur}

AG Gesundheitsökonomische Gesamtrechnungen der Länder (2014). Definitionen. [www.ggrdl.de/ggr_definitionen.html\#Gesundheitsmarkt].

Aronowitz, R. (2008). Framing-Effekte für soziale Verteilungsmuster von Krankheit: Ein unterschätzter Mechanismus. In: Niewöhner, J., C. Kehl, S. Beck (Hrsg), Wie geht Kultur unter die Haut? Emergente Praxen an der Schnittstelle von Medizin, Lebens- und Sozialwissenschaft. (S. 171-194). Bielefeld: transcript.

Bundesgesundheitsministerium (2014): Hermann Gröhe: „Krankheiten vermeiden, bevor sie entstehen ". Bundeskabinett beschließt Präventionsgesetz. Pressemitteilung Nr. 65 vom 17. Dezember.

Bundesregierung (2014). Entwurf eines Gesetzes zur Stärkung der Gesundheitsförderung und der Prävention. (Präventionsgesetz-PrävG).

[www.bmg.bund.de/themen/praevention/praeventionsgesetz.html].

Deloitte \& Touche GmbH (2014). Perspektive E-Health. Consumer-Lösungen als Schlïssel zum Erfolg? [www2.deloitte.com/de/de/pages/technology-media-andtelecommunications/articles/tmt-studie-perspektive-ehealth.html].

Deutsche Gesellschaft für Ernährung (DGE) e. V. im Auftrag des Bundesministeriums für Verbraucherschutz, Ernährung und Landwirtschaft (Hrsg.). (2004). Ernährungsbericht. Bonn: Autor.

Dietrich, A. (2011). Krankenversorgung als Hilfe zur Selbsthilfe? Risiken und Nebenwirkungen von Idealvorstellungen eigenverantwortlicher Patient/-innen für die Versorgungsgestaltung. Jahrbuch für kritische Medizin und Gesundheitswissenschaften. Verantwortung - Schuld - Sühne 46, 143-161.

Fachgruppe Ernährungs- und Verbraucherbildung (2005). Schlussbericht REVIS Modellprojekt. Reform der Ernährungs- und Verbraucherbildung in Schulen. [http://evbonline.de/evb_revis_schlussbericht.php].

Flexperto.com (Hrsg.). (2014). Der zweite Gesundheitsmarkt boomt - Healthstyle ist jetzt angesagt.

[www.blog.flexperto.com/wp-content/uploads/2014/05/Whitepaper-Der-zweiteGesundheitsmarkt-boomt-Healthstlye-ist-jetzt-angesagt-Flexperto.pdf].

Friedrichs, J. (2013). Das tollere Ich. Weniger schlafen, produktiver arbeiten, besser leben: Wie Menschen sich mithilfe der Technik selbst optimieren. Zeit online [www.zeit.de/2013/33/selbstoptimierung-leistungssteigerung-apps]. 


\section{Gesundheit und Selbstverantwortung}

Focus online (2015). Neuer Bluttest nutzt Smartphone-Akku. Kommt bald der HIVTest per App?

[www.focus.de/gesundheit/videos/neuer-bluttest-nutzt-smartphone-akku-kommtbald-der-hiv-test-per-app_id_4462709.html].

Hahn, D. (2011). Prinzip Selbstverantwortung? Eine Gesundheit für alle? Verschiebungen in der Verantwortung für Gesundheit im Kontext sozialer Differenzierungen. In: Jahrbuch für kritische Medizin und Gesundheitswissenschaften. Verantwortung - Schuld - Sühne 46, 29-50.

Hirschfelder, G., A. Ploeger, J. Rückert-John, G. Schönberger (Hrsg.) (2015). Was der Mensch essen darf. Ökonomischer Zwang, ökologisches Gewissen und globale Konflikte. Wiesbaden: Springer VS.

Hurrelmann, K. \& Franzkowiak, P. (2010). Gesundheit.

[www.bzga.de/leitbegriffe/?id=sysverz_liste_1\&idx=143].

Klafki, W. (1996). Grundzüge eines neuen Allgemeinbildungskonzepts. Im Zentrum: Epochaltypische Schlüsselprobleme. In W. Klafki (Hrsg.), Neue Studien zur Bildungstheorie und Didaktik. Zeitgemäße Allgemeinbildung und kritischkonstruktive Didaktik (5., unveränd. Aufl.; S. 43-81). Weinheim: Beltz.

Leutnant, S. (Hrsg.) (2014). Plan L. Leben bewusst gestalten. Ernährung Konsum Gesundheit. Paderborn: Schöningh Verlag.

Lifeline. Das Gesundheitsportal (2015). HIV-Test auf dem Smartphone. [www.lifeline.de/news/medizin-gesundheit/hiv-test-auf-dem-smartphoneid144272.html].

Nationaler Aktionsplan zur Prävention von Fehlernährung, Bewegungsmangel, Übergewicht und damit zusammenhängenden Krankheiten - IN FORM, [www.in-form.de].

Niewöhner, J. (2008): Die zeitlichen Dimensionen von Fett - Körperkonzepte zwischen Prägung und Lebensstil. In Niewöhner, J., C. Kehl, S. Beck (Hrsg.): Wie geht Kultur unter die Haut? Emergente Praxen an der Schnittstelle von Medizin, Lebens- und Sozialwissenschaft. (S. 113-142). Bielefeld: transcript.

Raacke, G. (2014). „Wer früher stirbt, ist selbst schuld!“ - Ein kritischer Blick auf das aktuelle Gesundheitsregime. Haushalt in Bildung \& Forschung, 3(4), 69-80.

rp-online (2015). Wie Sie die Mittagspause für Ihre Gesundheit nutzen.

[www.rp-online.de/leben/beruf/karriere/mittagspause-fuer-die-gesundheitnutzen-aid-1.4865634].

Schacky, v. C. (2008). Primary prevention of cardiovascular disease - how to promote healthy eating habits in population? Journal of Public Health, 16, 13-20.

Schlegel-Matthies, K. (2013). Ethik, Konsumentenverantwortung und Verbraucherbildung im Spannungsfeld. Haushalt in Bildung \& Forschung, 2(2), 61-70.

Schlegel-Matthies, K. (2015). Zwischen Wissenschaft und Lebenswelt. Entwicklung, Stand und Zukunftsperspektiven haushaltsbezogener Bildung. Paderborn 


\section{Gesundheit und Selbstverantwortung}

(erscheint als Band 10 der Paderborner Schriften zur Ernährungs- und Verbraucherbildung).

Schmidt, B. (2010). Der kleine Unterschied: Gesundheit fördern - und fordern. In B. Paul \& H. Schmidt-Semisch (Hrsg.), Risiko Gesundheit. Über Risiken und Gesundheit - das Maß aller Dinge? Nebenwirkungen der Gesundheitsgesellschaft (S. 23-37). Wiesbaden: VS Verlag für Sozialwissenschaften.

Sibbel, L. \& Kirchner, J. (2015). So gefährlich sind Fett, Salz, Zucker und Alkohol. Die Welt vom 9. Februar. [www.welt.de/137280281].

Statistisches Bundesamt (2014). Gesundheitsausgaben 2012 übersteigen 300 Milliarden Euro. Pressemitteilung 126/14 vom 7. April 2014.

Thaler, R. H. \& Sunstein, C. R. (2010). Nudge: Wie man kluge Entscheidungen anstößt. Berlin: Ullstein.

Tornieporth, G. (1977). Studien zur Frauenbildung. Ein Beitrag zur historischen Analyse lebensweltorientierter Bildungskonzeptionen, Weinheim Basel: Beltz.

Willett, W. C. \& Stampfer, M. J. (2013). Current evidence on healthy eating. Annual Review of Public Health, 34, 77-95.

[doi:10.1146/annurev-publhealth-031811-124646].

\section{Verfasserin}

Prof. ${ }^{\text {in }}$ Dr. Kirsten Schlegel-Matthies

Institut für Ernährung, Konsum und Gesundheit

Department Sport \& Gesundheit

Fakultät für Naturwissenschaften der Universität Paderborn

Warburger Str. 100

D-33098 Paderborn

E-Mail: kirsten.schlegel-matthies@upb.de

Internet: http://dsg.uni-paderborn.de/evb/personen/prof-dr-kirsten-schlegel-matthies 